\title{
Exploring the UV excess in star clusters of different mass
}

\author{
Fabiola Hernández-Pérez ${ }^{1}$ Gustavo Bruzual $^{2}$ and Gladis Magris C. ${ }^{3}$ \\ ${ }^{1}$ Universidad de Los Andes, Departamento de Física, Grupo de Astrofísica Teórica, Mérida, \\ Venezuela \\ email: fabiola.fchp@gmail.com, fabiolah@ula.ve \\ ${ }^{2}$ Centro de Radioastronomía y Astrofísica (CRyA), Morelia, México \\ email: $g$. bruzual@crya. unam.mx \\ ${ }^{3}$ Centro de Investigaciones de Astronomía (CIDA), Mérida, Venezuela \\ email: magris@cida.gob.ve
}

\begin{abstract}
We compute the expected spectral energy distribution of stellar populations of mass characteristic of star clusters taking into account stochastic fluctuations in the number of stars populating the IMF, and the presence of interacting binary stars in the cluster population. We evaluate under what circumstances the UV excess phenomenon is expected to appear in star clusters of different mass, and which is its most likely source: the stochastic fluctuations, the result of binary interactions, or a mixture of both.
\end{abstract}

Keywords. EHB stars, UV excess, star clusters.

\section{Introduction}

Observations of globular clusters (GCs) of M87 from Montes et al. (2014) show an UV excess that can not be explained by standard stellar population synthesis (SPS) models (e.g. Bruzual \& Charlot (2003), hereafter BC03). It is widely accepted that UV excess could be produced by an Extreme Horizontal Branch (EHB) component, probably due to binary interaction or He enhancement (see Smith et al. (2012)).

In this work we propose that stochastic sampling in the IMF could also drastically change the predictions of the models (with and without binary stars), explaining the UV bright GCs.

\section{Model and Results}

We use a recent implementation of $\mathrm{BC} 03$ model that allows a stochastic sampling of the initial mass function (IMF). Our test consists on 221 realizations of the Kroupa IMF, which is filled adding stars with stochastically selected mass, for a total mass of $10^{4}, 10^{5}$, $10^{6}$ and $10^{7} \mathrm{M}_{\odot}$. In Fig. 1 we present the resulting $\mathrm{UVr} 2 \mathrm{CD}$ for a $\mathrm{Z}=0.004$ population at 11 and 12 Gyr, Z=0.004 and Kroupa IMF. As we can see in Fig. 1, fluctuations in the IMF tend to spread the colours predicted by the model. The observations of GCs are covered by the bluest realizations (light gray dots), which are consequence of different numbers of central stars of planetary nebulae (CSPNe). We recall that BC03 model includes CSPNe as the main source of UV radiation in old populations.

In order to evaluate the influence of binary stars in the UV colours, we select some BC03 realizations of the IMF (white squares in left panel of Fig. 1) and use them as input in our SPS model (Hernández-Pérez \& Bruzual (2013), hereafter HB13) where EHB stars as product of binary interaction are included. To account for fluctuations in the number 


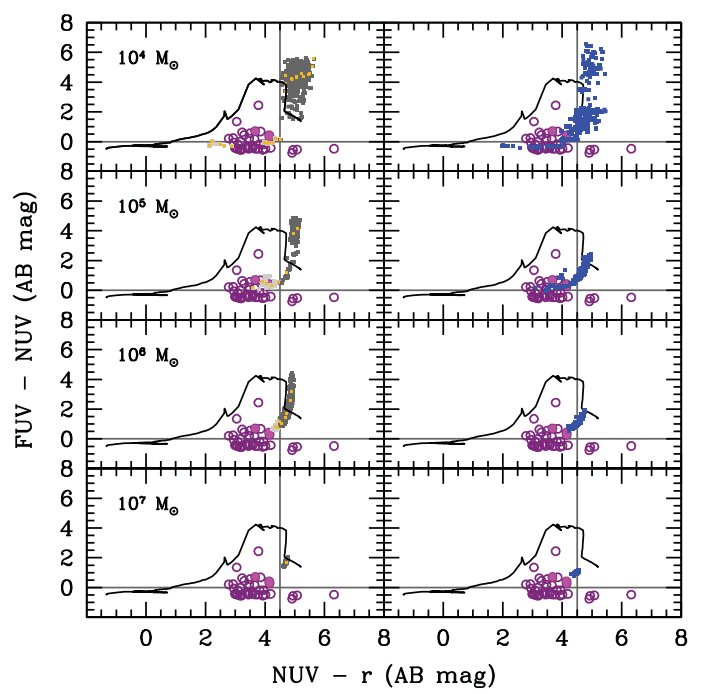

Figure 1. UVr2CD. Squares represent simulated clusters for standard (left panels) and binary (right panels) models. Light gray squares in left panels correspond to simulations with NUV-r $<4$.5. Standard model of infinite mass (solid black line) is also shown for comparison. Light gray open circles are M87 GCs from Montes et al 2014 with UV data, black filled triangles are those GCs with good UV photometry. See text for more details

of EHB progenitors, we run ten realizations of the binary related parameters of HB13 model for each white square realization of the IMF, with the same metallicity and ages. The resulting colours are represented as dark gray squares in the right panels of Fig. 1.

In a general way, the cloud of colours predicted by HB13 is similar that BC03, but binaries tend to shift the bulk of the cloud to the blue due to EHB stars. It is clear that observations of GCs, in particular those with good UV photometry (black filled triangles) are reproduced by both models, $\mathrm{BC} 03$ and $\mathrm{HB} 13$, if stochastic fluctuations in the IMF are included.

An extended analysis, including a consistent GC mass determination, and a more precise analysis of the colour distribution of GC in the UVr2CD, will help us to give a quantitative measure of the relevance of binaries to explain the UV excess in old populations.

\section{Conclusions}

- Standard model can not explain UV excess observed in GCs of M87.

- Stochastic fluctuations is the dominant source of UV excess predicted by both models for low mass populations.

- For higher masses, the increase in the number of CSPNe (BC03) and EHB (binary) tends to erase stochastic fluctuations.

- Binaries lightly shift the model to the blue in both NUV - r and FUV-NUV colours.

\section{References}

Bruzual, G. \& Charlot, S. 2003, MNRAS, 34, 100 (BC03)

Hernández-Pérez, F. \& Bruzual, G. 2013, MNRAS, 431, 2612 (HB13)

Montes, M., Acosta-Pulido, J., Prieto, M. \& Fernández-Ontiveros, J. 2014, MNRAS, 442, 1350

Smith, R., Lucey, J. \& Carter D. 2012, MNRAS, 421, 2982 